\title{
Les systèmes aquacoles face au changement climatique
}

\author{
Jérôme Lazard* \\ 21, Grand Rue, 34920 Le Crès, France
}

\begin{abstract}
Résumé - L'aquaculture contribue aujourd'hui pour environ $50 \%$ à l'approvisionnement en ressources aquatiques destinées à la consommation humaine et cette part est amenée à s'accroître à l'avenir compte tenu de la stagnation des captures liées à la pêche. Si un certain nombre de travaux ont été effectués en vue d'évaluer l'impact du changement climatique sur la pêche, peu a encore été fait dans ce domaine sur l'aquaculture. Cet article de synthèse tente d'identifier les défis auxquels l'aquaculture aura à faire face dans un contexte de changement climatique et propose des voies, à la fois adaptatives et innovantes, pour répondre à ces défis. L'article se focalise particulièrement sur six composantes de l'environnement susceptibles de subir des modifications sous l'effet du changement climatique et d'avoir un impact direct sur l'aquaculture : l'augmentation du niveau des mers ; la modification de la température ; les précipitations, les crues et les sécheresses ; la disponibilité en eau ; la dégradation de la qualité des eaux et enfin l'acidification des océans. Les impacts indirects concernent quant à eux principalement l'approvisionnement en farine et huile de poissons, constituants stratégiques des aliments destinés aux élevages d'animaux aquatiques, dont la disponibilité est dépendante des débarquements des pêches minotières, elles-mêmes sensibles au changement climatique. Face au changement climatique, deux stratégies sont possibles. La première, adaptative, consiste à mettre en œuvre des solutions qui permettent de prendre en compte les modifications du milieu (espèce adaptée, sélection de site) ; la deuxième consiste à imaginer des systèmes où les facteurs du milieu sont rigoureusement maîtrisés. Réciproquement, l'impact de l'aquaculture sur le changement climatique est évoqué. Enfin, les résultats d'une enquête conduite par la FAO en 2016 sur la situation des mesures prises dans diverses parties du monde pour faire face au changement climatique en matière d'aquaculture sont exposés.
\end{abstract}

Mots clés : aquaculture / changement climatique / impacts / adaptation / nouveaux systèmes d'élevage

\begin{abstract}
Aquaculture systems facing climate change. Aquaculture accounts today for approximately $50 \%$ of total aquatic products for human consumption. This contribution is expected to increase in the future taking into account the fact that capture fisheries are estimated to have reached their maximum level of catches. While some research has been carried out concerning the impact of climate change on capture fisheries, only very few has been done yet dealing with aquaculture. The present review paper aims at identifying the challenges aquaculture will need to face in this new context of climate change and at suggesting ways in order to forecast and overcome them. Six main components of the aquatic environment are investigated in view of their potential direct impact on aquaculture: sea level rise, changes in temperature, rainfall, river floods and droughts, water availability, water quality deterioration and ocean acidification. Indirect impact of climate change on aquaculture concerns mainly fish meal and oil as strategic inputs for feed manufacture, coming from pelagic fisheries whose catches are affected by climate change. Two strategies may be developed to address climate change challenges. The first one consists in developing solutions enabling to deal with the environmental changes induced by climate change (high salinity tolerant strain, site selection); the second consists in developing systems where all the environmental parameters are strictly monitored and controlled. Reciprocally, the impact of aquaculture on climate change is briefly mentioned. At the end of this paper, a comprehensive worldwide survey carried out by FAO in 2016 shows clearly that measures have already started to be implemented to deal with climate change in the field of aquaculture.
\end{abstract}

Keywords: aquaculture / climate change / impacts / adaptation / new culture systems

\footnotetext{
$\bar{*}$ Auteur de correspondance : jerome.lazard@cirad.fr
} 


\section{Introduction}

Une des plus grandes menaces générées par le changement climatique concerne l'impact que celui-ci est susceptible d'avoir sur les systèmes de production agricoles et donc sur l'alimentation humaine. Les prédictions dans ce domaine font état d'une réduction globale de la productivité agricole en région tropicale et subtropicale, qui constitue déjà une zone sensible de concentration de la population et de la pauvreté (IPCC, 2014).

Si l'étude de l'impact du changement climatique sur la production alimentaire a déjà fait l'objet de très nombreux travaux, il n'en est pas de même quant au secteur des ressources halieutiques qui n'a reçu jusqu'à récemment que relativement peu d'attention dans ce domaine (De Silva, 2012 ; Gros, 2014) avec une priorité consacrée à l'impact sur la biodiversité et les habitats marins (exemple des récifs coralliens). On estime que le secteur des pêches et de l'aquaculture subvient aux besoins d'environ 550 millions de personnes (soit $8 \%$ de la population totale) pour leurs moyens d'existence et leurs revenus ainsi que pour satisfaire leurs besoins nutritionnels en protéines (FAO, 2016). Allison et al. (2009) estiment que les populations potentiellement les plus affectées par l'impact du changement climatique sur le secteur halieutique sont situées dans les régions tropicales côtières, et particulièrement les zones de deltas et les pays insulaires en développement. Ces populations figurent déjà parmi les plus pauvres, deux fois plus dépendantes du poisson comme ressource alimentaire que dans les autres pays, avec $27 \%$ des besoins en protéines couverts par des aliments d'origine aquatique contre $13 \%$ pour le reste du monde (Tacon et al., 2010).

Le consensus général sur l'impact du changement climatique sur les pêches de capture est que les récents changements observés sur la distribution et la production d'un certain nombre d'espèces sont imputables à la variabilité du climat tel que le phénomène El Niño. Les prospectives récentes (Blanchard et al., 2012) tablent à l'horizon 2050 sur des changements de la production naturelle de poisson de l'ordre de $30 \%$, globalement en baisse dans les régions tropicales du fait de la réduction des upwellings (remontée d'eau froide riche en sels minéraux), et en hausse sous les hautes latitudes du fait du réchauffement des eaux et de la réduction de la couverture de glace. Les pêcheries continentales qui contribuent pour $13 \%$ aux captures totales pourraient être également affectées négativement par une modification (à la baisse) des précipitations dans certaines régions. Jusqu'à présent, il n'y a eu que relativement peu d'impact du changement climatique sur l'aquaculture (De Silva et Soto, 2009). Ce secteur de production a connu une croissance spectaculaire depuis les années $1980(+10 \% /$ an) et représente aujourd'hui une part essentielle de la fourniture de produits d'origine aquatique pour l'alimentation humaine. En 2014, la contribution de l'aquaculture hors plantes aquatiques s'élevait à 74 millions de tonnes (Mt) sur un total pêche + aquaculture de $167 \mathrm{Mt}$ (FAO, 2016).

Cet article a pour objectif d'évaluer les défis à relever par l'aquaculture face aux modifications que le changement climatique imposera à cette activité. Les principales composantes du changement climatique susceptibles d'affecter l'aquaculture seront envisagées, de même que leur impact sur celle-ci. La région Asie-Pacifique domine la production aquacole mondiale avec $90 \%$ de la production totale (FAO, 2016) : elle constituera donc l'essentiel des références présentées ici. Par ailleurs, il convient de noter que seul un petit nombre d'études de cas concrets de l'impact du changement climatique sur l'aquaculture en tant que telle a été réalisé et est donc disponible.

L'aquaculture se différencie des activités agricoles par le nombre d'espèces d'élevage qui a augmenté à un rythme élevé ces 20 dernières années. En 2012, la FAO (2014) en dénombrait plus de 500 , dont 350 espèces de poissons, sachant que 15 d'entre elles représentaient $75 \%$ de la production totale. Ces espèces sont élevées dans une grande diversité de systèmes de production aquacole à divers niveaux d'intensification, en eaux douces, saumâtres et de mer. Pour la plupart des espèces aquatiques, des technologies d'écloserie fiables sont disponibles et permettent la production massive de juvéniles des différentes espèces. Seul l'élevage de quelques espèces reste inféodé à la collecte d'alevins dans le milieu naturel (anguille, certains mollusques).

L'aquaculture est pratiquée à travers une vaste étendue géographique et climatique. Si les activités aquacoles actuelles, basées sur les quatre principaux groupes d'espèces (poissons, mollusques, crevettes et plantes aquatiques), sont développées à toutes les latitudes, l'essentiel de la production est concentré en Asie et dans les pays tropicaux.

\section{Impacts potentiels du changement climatique sur l'aquaculture}

Les impacts du changement climatique se manifestent sous diverses formes : ils peuvent être directs ou indirects et certains d'entre eux peuvent agir en synergie ou secondairement (par exemple salinisation des deltas suite à l'augmentation du niveau des océans). Les diverses composantes de l'environnement susceptibles de subir des modifications sous l'effet du changement climatique, et donc d'avoir un impact sur l'aquaculture, sont présentées sur la Figure 1.

Ont été retenus dans cet article les facteurs de l'environnement qui nous ont paru avoir (ou susceptibles d'avoir dans l'avenir) les impacts les plus significatifs sur l'aquaculture.

\section{Principaux défis de l'aquaculture face au changement climatique}

\subsection{Impacts directs}

\subsubsection{Augmentation du niveau de la mer}

L'augmentation du niveau de la mer se fera sentir au niveau des régions côtières et deltaïques et les conséquences seront tout particulièrement génératrices de conséquences en zone tropicale où elles correspondent à des zones d'activités agricoles intenses (y compris l'aquaculture).

Les impacts de la montée du niveau des eaux se traduiront par la salinisation et les inondations, notamment dans les deltas. Des zones telles que le delta du Mékong, la région de Cà Mau au Sud-Viêt Nam et le delta du Gange-Brahmapoutre au Bangladesh, où l'élevage de la crevette en alternance avec 


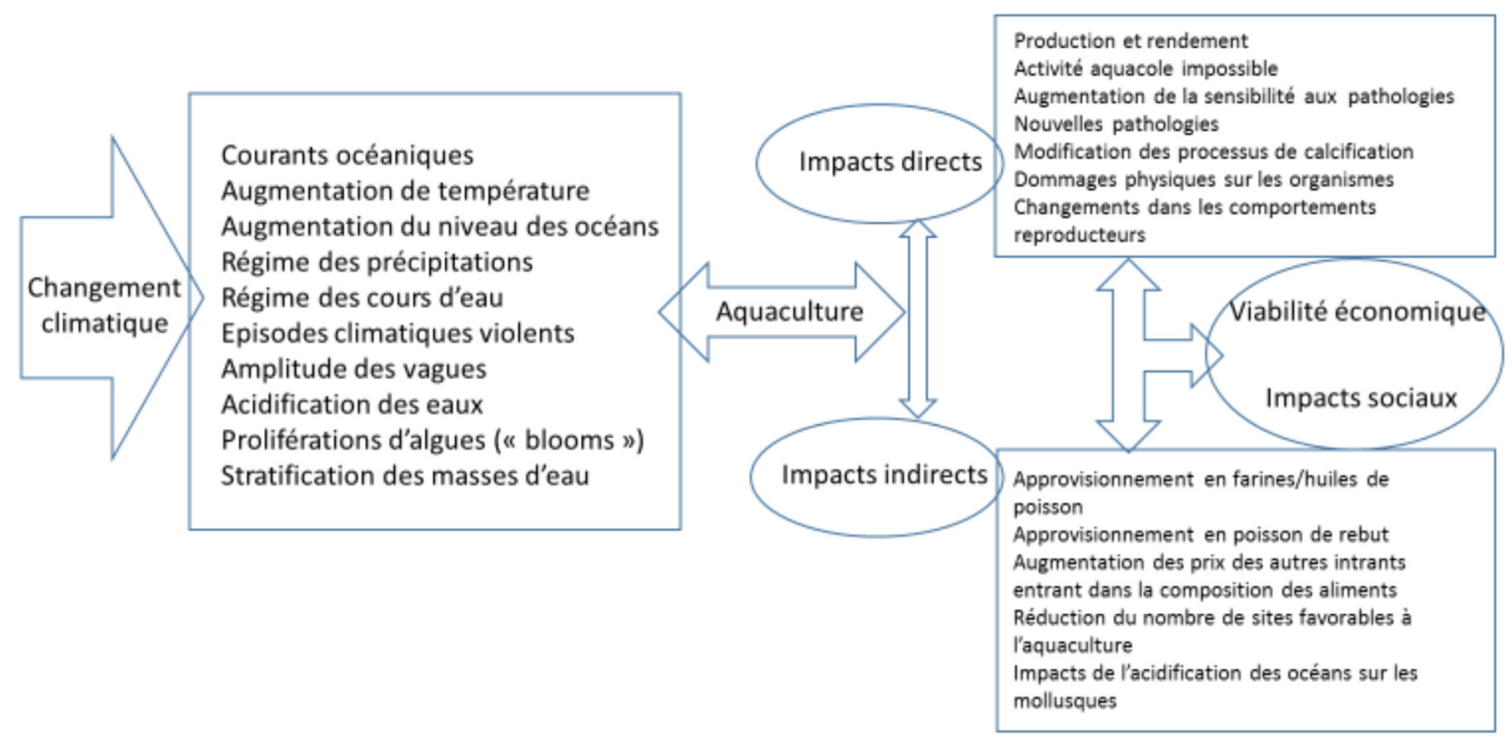

Fig. 1. Représentation schématique des impacts potentiels du changement climatique sur l'aquaculture et les différentes formes de leur manifestation (d'après De Silva, 2012).

Fig. 1. Schematic representation of the potential major climate change impacts on aquaculture and the possible forms of their manifestation (after De Silva, 2012).

la riziculture irriguée constituent l'essentiel de l'activité agricole, seront particulièrement affectées.

La montée du niveau des eaux de mer rendra plus difficile l'évacuation des crues des fleuves, de même que la gestion de l'eau des bassins d'élevage de crevettes et tout particulièrement leur vidange. De nouveaux plans d'aménagement et de gestion de l'eau devront être mis en place, qui sont susceptibles de provoquer des conflits entre les divers utilisateurs des terres concernées (Tan, 2008).

Les difficultés rencontrées pour évaluer l'impact de l'augmentation du niveau de la mer sur les activités aquacoles sont largement illustrées par l'exemple emblématique de la filière de production du poisson-chat Pangasianodon hypophthalmus dans le delta du Mékong. Cette filière, qui représente un volume de production de $1,2 \mathrm{Mt}(60 \%$ de la production aquacole totale du Viêt Nam), emploie 500000 personnes et génère 1,5 milliards de $\$$ de devises (Anh, 2014). Le système aquacole actuel repose sur un échange d'eau régulier entre les étangs (qui couvrent une superficie totale de 5600 ha) et le fleuve, qui autorise des densités très élevées de poisson et des niveaux de productivité parmi les plus élevés obtenus dans le monde en pisciculture, de 300 à $400 \mathrm{t} / \mathrm{ha} /$ cycle (Lazard et al., 2009). Les deux paramètres pris en compte jusqu'à présent pour l'implantation des étangs d'élevage ont principalement été le niveau des crues en saison des pluies et la limite d'influence de la salinité en saison sèche, des concentrations supérieures à $4 \mathrm{~g} / \mathrm{L}$ étant considérées comme impropres à l'élevage de ce poisson (De Silva et Phuong, 2011). Un accroissement du niveau de la mer de $1 \mathrm{~cm} / \mathrm{an}$ jusqu'en 2100 (Grinsted et al., 2009), couplé à une réduction de l'intensité des crues, provoquera une augmentation de la pénétration d'eaux salées durant la saison sèche, ainsi qu'un plus grand épandage des crues durant la saison des pluies en amont. Une modélisation effectuée par Anh (2014) selon trois scénarios - niveaux de la mer : $+30 \mathrm{~cm},+50 \mathrm{~cm}$ et $+75 \mathrm{~cm}$ par rapport à 2005 - met en évidence une expansion des surfaces en eaux salées de salinité supérieure à $4 \mathrm{~g} / \mathrm{L}$ du delta du Mékong de 2, 3 et $7 \%$ respectivement. L'augmentation des surfaces correspondant à une salinité comprise entre 10 et $20 \mathrm{~g} / \mathrm{L}$ serait de 14,11 et $13 \%$ respectivement. Ce phénomène s'accompagnera de dynamiques hydrologiques entraînant une redistribution géographique des zones affectées par la salinité. Certaines zones verront leur salinité augmenter et d'autres se réduire, comme par exemple dans ce dernier cas lorsque l'eau douce fluviale poussée par la langue salée se déverse dans des zones de niveau topographique inférieur. L'impact de ces évolutions au niveau des activités aquacoles sera donc éminemment variable selon les sites où sont implantées les infrastructures d'élevage, certaines allant même jusqu'à en bénéficier. Des cartographies plus précises pour affiner les prospectives seront nécessaires pour définir le statut des exploitations aquacoles actuelles et futures vis-à-vis des variations de salinité (Halls et Johns, 2013).

La limite de tolérance à la salinité de $P$. hypophthalmus, fixée à $4 \%$, est remise en cause par des travaux récents. Nguyen et al. (2011), testant l'élevage de juvéniles de $25 \mathrm{~g}$ à des salinités croissantes $(0$ à $15 \mathrm{~g} / \mathrm{L})$, ont mis en évidence la bonne survie de cette espèce mais une croissance réduite à $9 \mathrm{~g} / \mathrm{L}$; alors que Castaneda et al. (2010) rapportent une croissance non affectée jusqu'à une salinité de $13 \mathrm{~g} / \mathrm{L}$. Do et Tran (2012) ont quant à eux montré que les œufs de $P$. hypophthalmus pouvaient être incubés à une salinité de $11 \mathrm{~g} / \mathrm{L}$. Ces résultats peuvent être considérés comme le signe de la capacité de l'espèce à s'adapter à la salinité et à répondre positivement à des programmes de sélection dans ce sens.

Parmi les stratégies disponibles pour faire face au défi de l'augmentation de la salinité des eaux d'élevage figure celle consistant à développer une souche de cette espèce de poissonchat tolérante à la salinité et à assurer sa diffusion auprès des pisciculteurs. Outre le fait que cette option exige de mettre en 


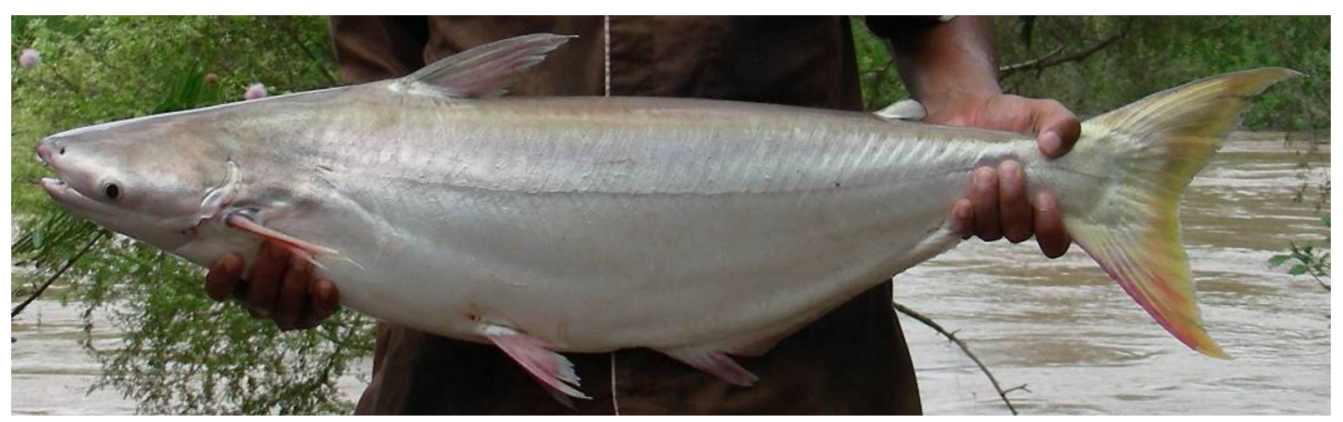

Fig. 2. Pangasius krempfi : une espèce euryhaline de poisson-chat du Mékong, d'intérêt aquacole potentiel pour l'élevage en eaux saumâtres. Fig. 2. Pangasius krempfi: an euryhalin Mekong catfish species of potential aquaculture interest for culture in brackish waters.

Tableau 1. Tolérance de quelques espèces piscicoles d'élevage des principales zones climatiques à divers niveaux de température (adapté d'après Ficke et al., 2002).

Table 1. Temperature tolerances of selected cultured species of different climate distribution (adapted after Ficke et al., 2002).

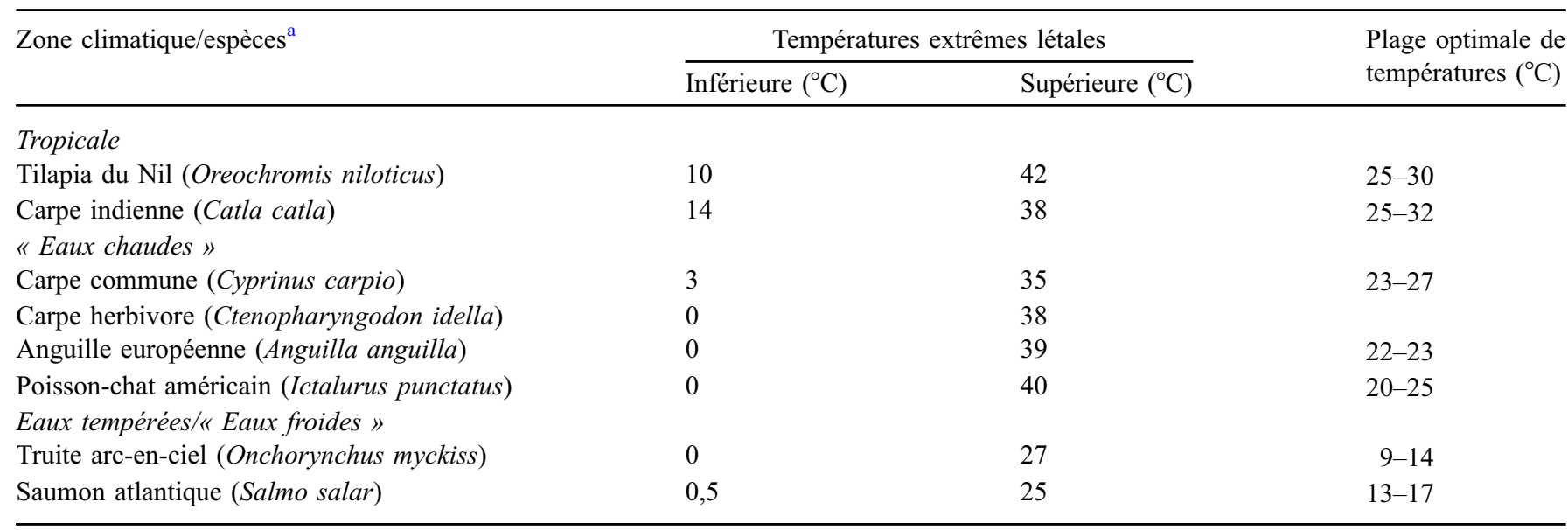

a Toutes les espèces exposées dans ce tableau font partie des 15 les plus produites au monde en 2014 (FAO, 2016).

place un programme de sélection génétique requérant technicité, infrastructures et temps, elle n'est pas sans danger en termes d'impact sur la biodiversité des espèces naturelles (sauvages). Une solution plus respectueuse de l'environnement consisterait à domestiquer des espèces indigènes de Pangasiidae vivant naturellement dans l'estuaire du fleuve et qui seraient donc bien adaptées à la pisciculture en eau saumâtre. C'est le cas en particulier de deux espèces, Pangasius krempfi et Pangasius mekongensis. Les premiers essais d'élevage conduits sur P. krempfi (Fig. 2) ont permis d'établir les conditions préliminaires en vue de sa reproduction en captivité (Clota, 2006 ; Cacot et Lazard, 2009).

\subsubsection{Modification de la température}

Contrairement aux autres animaux d'élevage, tous les animaux aquatiques utilisés en aquaculture sont poikilothermes (température corporelle qui varie avec celle de leur milieu). En conséquence, toute modification de température de leur environnement a un impact sur le métabolisme global et donc sur la vitesse de croissance et sur le rendement final, sur la saisonnalité de la reproduction ainsi que sur le rythme des pontes et la fécondité, sur la sensibilité aux pathogènes et aux substances toxiques. Les températures létales, supérieure et inférieure, et la température optimale varient considérablement en fonction des espèces. Toute modification du climat et donc de la température aura un impact sur les activités aquacoles relatives à une espèce donnée (Tab. 1).

Les moyens d'affronter ces modifications de l'environnement consistent à élever des espèces tolérant des températures plus élevées, à développer des souches de la même espèce tolérantes à des plus hautes températures, à mettre au point des systèmes d'élevage en circuit fermé dans lesquels les paramètres environnementaux peuvent être contrôlés. La sélection de souches de saumons tolérant des températures plus élevées que celles auxquelles elles sont accoutumées a déjà été amorcée (Fish Farmer, 2008) et le développement de circuits fermés thermorégulés (voir Sect. 5) est déjà largement répandu.

L'augmentation de la température dans les régions tempérées a provoqué une aggravation de la virulence de certains organismes pathogènes qui étaient relativement inoffensifs aux températures ordinairement tolérées par les espèces d'élevage. Ces changements de température ont, par exemple, provoqué l'accroissement de la virulence de pathogènes tels que Vibrio harveyi, occasionnant des mortalités élevées sur les ormeaux (Travers et al., 2009). Dans le cas du tilapia Oreochromis niloticus, une plus grande 
pathogénicité du Streptococcus agalactie, agent responsable majeur en Asie et ailleurs de la maladie, ainsi qu'une plus forte réaction inflammatoire (délétère) ont été observées aux températures plus élevées (Kayansamruaj et al., 2014 ; Marcusso et al., 2015).

\subsubsection{Précipitations, crues et sécheresses}

L'eau douce est généralement considérée comme la ressource naturelle la plus en danger dans une perspective de changement climatique. Dans la mesure où l'essentiel de la production aquacole s'effectue en eaux continentales (douces et saumâtres), dont $90 \%$ sur le continent asiatique, et où ce continent dispose des plus faibles ressources en eau douce par habitant, une attention toute particulière devra être portée sur son usage, notamment pour son utilisation en aquaculture (voir Sect. 4).

Ainsi, les changements de régime des saisons des pluies (moussons) et particulièrement leur raréfaction, vont-ils modifier les pratiques aquacoles existantes ? Par exemple, pour la plupart des espèces tropicales, la période de reproduction intervient durant la saison des pluies, y compris dans le cas des écloseries dont les géniteurs sont maintenus dans la majeure partie des cas dans des infrastructures (étangs) extérieures (Thackery et al., 2010). Par ailleurs, l'approvisionnement en géniteurs est fréquemment assuré à partir de populations du milieu naturel. Ainsi, il a été mis en évidence que les changements de régime des pluies et donc des crues ont décalé les saisons de reproduction des carpes indiennes (Labeo rohita, Catla catla et Cirrhinus mrigala) dans le milieu naturel avec des conséquences sur la saisonnalité de la production d'alevins en écloserie (Vass et al., 2009).

\subsubsection{Disponibilité globale en eau}

L'eau douce, qui héberge $75 \%$ de la production aquacole mondiale (hors plantes aquatiques), est disponible en quantité limitée sous forme de rivières, lacs, zones humides, etc. Elle ne représente que $0,01 \%$ des ressources aquatiques terrestres totales, soit $113000 \mathrm{~km}^{3}$. En outre, elle est inégalement répartie selon les continents, avec des écarts prononcés lorsqu'elle est exprimée en disponibilité par habitant : 3,9 $\mathrm{m}^{3} /$ hab/an en Asie, 4,2 en Europe, 5,7 en Afrique, 17,4 en Amérique centrale et du Nord, 38,2 en Amérique du Sud et 82,2 en Australie et Océanie (Nguyen et De Silva, 2006). Jusqu'à présent, les besoins actuels et futurs en eau douce de l'humanité ont principalement été calculés en prenant en compte la consommation de l'agriculture et, sur ces bases, le déficit en eau douce à l'horizon 2050 a été estimé à $3800 \mathrm{~km}^{3} / \mathrm{an}$ (Falkernmark et al., 2009). Les besoins pour l'aquaculture n'ont que très accessoirement été pris en compte, mais dans un tel contexte, les systèmes aquacoles à développer à l'avenir devront être économes en eau (voir Sect. 5).

\subsubsection{Désoxygénation, stratification des eaux et efflorescences algales}

La synthèse de Gros (2014) souligne que depuis les années 1950, l'océan a stocké plus de $90 \%$ de l'augmentation de la quantité de chaleur reçue par la planète, soit une contribution au bilan thermique du système Terre très supérieure à celles de ses autres composantes (continents, atmosphère, calottes glaciaires et banquise). Gros (2014) cite Levitus et al. (2012) qui estiment qu'entre 1955 et 2010 , la température moyenne a augmenté de $0,18^{\circ} \mathrm{C}$ dans la couche $0-700 \mathrm{~m}$ de l'océan, renforçant la stratification des eaux superficielles (moins denses parce que réchauffées, elles se mélangent moins avec les eaux plus profondes). Ce dernier phénomène est l'une des causes de la désoxygénation de l'océan (moins d'O $\mathrm{O}_{2}$ transféré en profondeur depuis la surface). Combiné à l'augmentation de température qui diminue la solubilité de 1 ' $\mathrm{O}_{2}$, il entraîne l'expansion des zones hypoxiques (Seibel, 2011). À ces changements observés au large - dans le Pacifique nord et dans les zones tropicales appauvries en $\mathrm{O}_{2}-\mathrm{s}$ 'ajoutent les effets de l'eutrophisation des eaux côtières, due aux apports de sels nutritifs en provenance des bassins versants et de l'atmosphère. À l'échelle de l'océan, qui perdrait 1 à $7 \%$ de son contenu en $\mathrm{O}_{2}$ au cours de ce siècle, l'étude de la désoxygénation - qui n'est appréhendée que depuis une décennie - ne fait que commencer (Keeling et al., 2010).

Historiquement, l'aquaculture s'est essentiellement développée à terre, avec pour principale structure d'élevage l'étang (bassin en terre), qui représente encore aujourd'hui environ $70 \%$ de la production de poisson d'eau douce et $100 \%$ de celle de crevette. Néanmoins, un nouveau système s'est développé à grande échelle depuis les années 1980, celui des cages flottantes dans divers milieux aquatiques : en mer, dans les fleuves, les lacs naturels et les lacs de barrages. De nombreux spécialistes (Ficke et al., 2007 ; Alric, 2006) estiment que le changement climatique risque d'exacerber l'eutrophisation des divers plans d'eau avec prolifération d'organismes phytoplanctoniques et d'entraîner une stratification thermique accrue, particulièrement dans les zones tropicales. Une eutrophisation accrue provoquera une chute de l'oxygène dissous en début de journée. Des perturbations atmosphériques (pluies, refroidissement de la surface de l'eau sous l'action du vent) sont susceptibles de provoquer un retournement des eaux les plus profondes, désoxygénées et riches en gaz toxiques tels que $\mathrm{H}_{2} \mathrm{~S}$, qui, arrivant en surface, provoquent d'importantes mortalités dans les couches d'eau supérieures où sont implantées les cages flottantes. De même en mer, le réchauffement climatique associé à l'eutrophisation du milieu provoque la prolifération d'algues planctoniques (algal blooms) qui peuvent dans certaines conditions former des « marées » rouges ou vertes dont les conséquences peuvent être des mortalités massives de poissons et des intoxications alimentaires chez les consommateurs de mollusques élevés dans cet environnement.

De façon générale, l'élevage en cage s'est considérablement intensifié ces dernières années, à la fois en termes de densité de stockage en poissons par unité de volume et de nombre de cages dans les milieux d'élevage. Une implantation optimale des cages devrait privilégier les espaces aquatiques disposant du niveau maximum de circulation et de renouvellement d'eau au détriment de la proximité des berges, plus pratique sur le plan logistique. Les autorités gestionnaires des plans d'eau devraient être attentives, en termes de gestion globale de ceux-ci, à faire participer les responsables de fermes aquacoles pour définir des zones optimales d'implantation des cages, ainsi que des normes de densité de stockage et d'alimentation. La conduite d'une évaluation de la capacité d'accueil (carrying capacity) des différents plans d'eau utilisés pour l'aquaculture en cages flottantes doit permettre de gérer 
au mieux l'implantation de nouvelles fermes aquacoles et d'éviter les accidents liés à de brutales anoxies et remontées de substances toxiques à partir du fond (ce travail vient, par exemple, d'être réalisé pour le lac Volta au Ghana (Asmah et al., 2015). Dans certains cas, les gestionnaires de barrages peuvent avoir recours à la « biomanipulation » des plans d'eau (Lazzaro et Starling, 2005), par l'introduction d'espèces agissant sur la cascade trophique ou d'espèces phytoplanctonophages agissant directement sur la réduction de l'abondance du phytoplancton et par là même des risques d'eutrophisation de la retenue. Les espèces introduites constituent également un moyen d'augmenter la productivité du plan d'eau et bénéficient directement aux pêcheurs, qui constituent la population la plus défavorisée par le développement à grande échelle des fermes aquacoles. Ces opérations doivent cependant être effectuées avec beaucoup de vigilance. Elles doivent tout d'abord privilégier l'utilisation d'espèces indigènes/autochtones autant que possible et, dans le cas où les gestionnaires ont recours à des espèces exotiques/exogènes (en général la carpe argentée), assurer un suivi de l'impact de celles-ci sur l'écosystème concerné. En Israël, par exemple, le lac Kinneret fut intensivement empoissonné en carpe argentée durant les années 1970 et 1980 pour contrôler le développement du phytoplancton, mais dans cet environnement, ce poisson s'est révélé alternativement phytoplanctivore et zooplanctivore en fonction des saisons, et ce comportement a contribué à réduire la consommation de phytoplancton par le zooplancton au moment de l'année où les risques de développement de blooms algaux sont les plus grands. Suite à ce constat, l'empoissonnement de ce lac en alevins de carpes argentées a été interrompu (Spataru et Gophen, 1985).

\subsubsection{Acidification des océans}

Depuis le début de l'ère industrielle, près de la moitié du $\mathrm{CO}_{2}$ anthropogénique s'est accumulée dans l'atmosphère et contribue à l'effet de serre, tandis qu'un quart de ce $\mathrm{CO}_{2}$ a été absorbé par l'océan (Sabine et Tanhua, 2010). En piégeant une partie des émissions anthropiques, l'océan atténue le changement climatique mais la dissolution du $\mathrm{CO}_{2}$-outre qu'elle est en voie d'affaiblir de $30 \%$ la capacité de l'océan à absorber ce gaz - a entraîné deux modifications majeures des propriétés chimiques des eaux de surface :

- un accroissement de $34 \%$ de leur concentration en ions hydrogène $\mathrm{H}^{+}$, mesuré par l'abaissement du $\mathrm{pH}(8,2$ en $1850,8,1$ aujourd'hui et vraisemblablement 7,8 en 2100); - une diminution de $16 \%$ de leur concentration en ions carbonate $\mathrm{CO}_{3}{ }^{2-}$, mesurée par la diminution de la saturation en carbonates (Gros, 2014).

Les eaux océaniques superficielles, aujourd'hui sursaturées en carbonates, créent des conditions favorables à la précipitation du $\mathrm{CaCO}_{3}$ sous forme d'aragonite (un polymorphe stable à haute pression et haute température du $\mathrm{CaCO}_{3}$, lequel rentre dans la composition de la coquille des huîtres) ou de calcite qui entrent dans la composition des pièces calcifiées de nombreux organismes marins et notamment d'élevage. D'ici à quelques décennies, du fait de l'acidification, une grande partie des eaux de surface sera sous-saturée en aragonite dans l'océan Austral et sous les hautes latitudes de l'hémisphère nord, et inexorablement, le volume de l'habitat océanique favorable à la calcification des mollusques sauvages et cultivés sera rétréci.

Le déficit de production de naissain d'huîtres creuses d'une écloserie de la côte de l'Oregon pendant les périodes d'upwelling intense fournit un premier exemple d'impact de l'acidification sur l'ostréiculture. Dans cette région, l'upwelling apporte en surface des eaux sous-saturées en aragonite, matériau de la coquille des larves d'huîtres. Compte tenu des prévisions d'accentuation de la sous-saturation (Gruber et al., 2012), l'entreprise a choisi d'installer une nouvelle écloserie à Hawaii.

Le second exemple concerne l'huître creuse du Pacifique dont les performances reproductrices ont été affectées ces dernières années dans l'État de Washington (États-Unis). Ce problème de reproduction constaté en 2004 a affecté non seulement les stocks d'huîtres naturels, mais également les stocks domestiqués dans les écloseries qui utilisent la même source d'eau de mer. Les travaux conduits pour comprendre les mécanismes de ce phénomène ont révélé que l'impact de l'acidification est spécifique à l'espèce. Par exemple, il a été démontré que les larves de deux espèces phylogénétiquement proches de l'huître creuse du Pacifique étaient très sensibles à la concentration de $\mathrm{CO}_{2}$, c'est-à-dire à la diminution du $\mathrm{pH}$. Par contre, les huîtres de Suminoe, natives du Pacifique ouest, ne sont pas affectées par l'acidification des eaux océaniques (De Silva et Soto, 2009).

\subsection{Impacts indirects}

Les impacts indirects du changement climatique sur les activités aquacoles concernent principalement la disponibilité en farine et huile de poissons pour l'incorporation dans les aliments destinés aux élevages d'animaux aquatiques.

En 2012, l'aquaculture absorbait $70 \%$ de la farine de poisson et $75 \%$ de l'huile de poisson extraites des pêches minotières (pêches dévolues à la capture d'espèces, principalement des petits pélagiques, destinées à la fabrication de farine et d'huile de poisson) (Tacon et Metian, 2015). Il convient cependant de noter que malgré l'augmentation mondiale de la demande en poisson pour l'alimentation humaine, la proportion de poisson utilisée pour des usages non alimentaires est restée constante depuis 1970 (25-30\%).

Pour ce qui concerne spécifiquement l'évolution future des pêches minotières dans un contexte de changement climatique, la gestion des plus grandes pêcheries, péruviennes en tête, a démontré son fort niveau de robustesse et de résilience aux fluctuations environnementales et au niveau élevé de la demande en farine/huile de poisson (Arias-Schreiber et al., 2011). Autrement dit, un niveau soutenu d'exploitation de ces pêcheries de petits pélagiques supporterait une forte demande en huile et farine, contrairement à ce que soutiennent divers autres auteurs (Smith et al., 2011) qui recommandent de gérer ces stocks en dessous du maximum sustainable yield (MSY).

$\mathrm{Si}$ l'aquaculture doit poursuivre sa contribution à l'alimentation humaine dans un contexte de croissance démographique et de stagnation des ressources halieutiques au niveau de 90-100 Mt, elle devra produire un volume supplémentaire de $70 \mathrm{Mt}$ par rapport à 2014 pour nourrir 9,3 milliards d'habitants à l'horizon 2050 de façon durable (Merino et al., 2012). Des adaptations technologiques seront 
alors nécessaires pour produire plus avec moins d'impact environnemental. Le poisson utilisé dans l'aliment aquacole pour produire une unité de produit aquatique (fish in-fish out [FIFO]) devra être réduit de $50 \%$ par rapport aux niveaux couramment utilisés aujourd'hui. Idéalement, les poissons pélagiques utilisés actuellement pour la fabrication de farine et d'huile de poisson devront être destinés à la consommation humaine ou à l'alimentation des espèces aquacoles dont le FIFO est le plus faible.

Des alternatives existent qui permettent d'ores et déjà de réduire la dépendance de l'aquaculture vis-à-vis des intrants issus des pêches minotières. Parmi celles-ci, on peut citer l'utilisation en élevage d'espèces à chaîne alimentaire courte, l'utilisation des prises accessoires et de sous-produits des usines de transformation et de conditionnement des produits de la pêche, l'utilisation d'espèces marines sans valeur marchande pour la consommation humaine pour la fabrication de farine de poisson, l'utilisation de micro-algues comme ingrédients des aliments composés pour l'aquaculture (Tacon et al., 2011). L'incorporation de produits d'origine végétale dans les aliments pour poisson (protéagineux, céréales, tourteaux d'oléagineux, etc.) en substitution aux intrants d'origine marine (Médale et al., 2013) déplace quant à lui le problème des écosystèmes marins vers les écosystèmes terrestres. La production de ces matières premières entre en compétition vis-à-vis des autres filières qui s'en approvisionnent, à commencer par la consommation humaine et l'agro-industrie. Cette évolution devra s'accompagner de l'élaboration de nouveaux outils d'évaluation des performances des systèmes aquacoles (voir Sect. 4).

\section{Impacts de l'aquaculture sur le changement climatique}

L'analyse des résultats de travaux conduits pour mesurer le coût énergétique des différentes filières de production animale, malgré la disparité des méthodologies et des données, met en évidence quelques tendances fortes. L'élevage d'organismes aquatiques à chaîne alimentaire longue se caractérise par un faible ratio protéine produite/énergie consommée, plus faible que celui de l'élevage d'animaux terrestres tels que le poulet, le porc et même le bœuf. D'un autre côté, l'élevage d'animaux aquatiques à chaîne alimentaire courte (carpes, tilapia) ou filtreurs (mollusques) présente un bilan énergétique favorable (Tab. 2).

Un autre type d'approche, plus holistique, permet de comparer différents systèmes de production aquacole en fonction de leur impact sur l'environnement : il s'agit de l'analyse de cycle de vie (ACV).

À titre d'exemple, six systèmes d'élevage aquacole fortement différenciés, du plus extensif au plus intensif, dans des pays développés et en développement, ont été évalués en fonction de leur impact environnemental en utilisant la méthode de l'ACV sur la base de cinq types d'impact (Lazard et al., 2014). Le score final est contre-intuitif puisque l'on s'attendrait à voir les systèmes extensifs présenter la meilleure durabilité environnementale alors que c'est l'inverse qui se produit. Cette situation étonnante s'explique par le fait que les systèmes intensifs présentent un niveau de capacité à valoriser les intrants et une efficience écologique supérieur à celui des systèmes extensifs.
Tableau 2. Classification de divers types d'élevage en fonction du ratio énergie protéique comestible/énergie industrielle consommée (d'après Tyedmers et Pelletier, 2007).

Table 2. Ranking of selected foods by ratio of edible protein energy (PE) output to industrial energy (IE) inputs, expressed as a percentage (after Tyedmers and Pelletier, 2007).

\begin{tabular}{ll}
\hline Type d'élevage & $\begin{array}{l}\% \text { énergie } \\
\text { protéique } \\
\text { produite/ } \\
\text { énergie } \\
\text { industrielle } \\
\text { consommée }\end{array}$ \\
& $100-111$ \\
\hline Élevage extensif de carpe, eau douce & 25 \\
Élevage intensif de poulet, États-Unis & 13 \\
Élevage extensif de tilapia, eau douce, Indonésie & $10-5$ \\
Élevage de moules, Scandinavie & 6,0 \\
Élevage intensif de tilapia, eau douce, Zimbabwe & 5,0 \\
Élevage bovin, pâture, États-Unis & 2,5 \\
Élevage bovin, feed lot, États-Unis & 2,5 \\
Élevage intensif de saumon atlantique, cage, Canada & 2,0 \\
Élevage de crevettes, semi-intensif, Colombie & 1,4 \\
Élevage de crevettes, intensif, Thaïlande & \\
\hline
\end{tabular}

Ces résultats en apparence contradictoires mettent en évidence la nécessité, pour caractériser l'efficience des systèmes aquacoles, de coupler diverses méthodes d'évaluation.

\section{Nouveaux systèmes d'élevage}

Dans un contexte de changement climatique, la stratégie consistera soit à s'adapter aux nouvelles conditions environnementales, soit à imaginer des systèmes qui s'en affranchissent. Ces « nouveaux » systèmes ont déjà commencé à émerger : ils ont pour caractéristique d'économiser (par l'optimisation de leur utilisation) certains des facteurs de production dont la raréfaction est prévisible, tels que l'eau douce et les protéines d'origine aquatique entrant dans la composition de l'aliment (Klinger et Naylor, 2012).

Le système qui sera évoqué ici est le système d'élevage en eau recirculée (RAS), qui permet à la fois une maîtrise complète des paramètres de la qualité de l'eau d'élevage, son intensification et une économie d'eau par une recirculation continue de l'eau d'élevage. Les RAS peuvent concerner des infrastructures implantées en plein air ou sous abri, et des étangs aussi bien que des bassins. La conception des systèmes peut varier, mais tous suivent le même principe de traitement de l'eau : élimination des résidus solides, élimination des nutriments ou détoxication (au moyen d'un filtre biologique hébergeant les bactéries nitrifiantes), élimination du $\mathrm{CO}_{2}$, supplémentation en oxygène dissous, stérilisation des bactéries et des organismes pathogènes de façon à ce que l'eau puisse être recyclée dans le système.

Les RAS, qu'ils soient d'eau de mer ou d'eau douce, présentent de nombreux avantages sur les systèmes aquacoles conventionnels, à commencer par l'économie d'eau et 
d'espace. Un système en eau recirculée en eau douce consomme $50 \mathrm{~L} / \mathrm{kg}$ de poisson produit, un système en eau recirculée en eau de mer reconstituée consomme $16 \mathrm{~L} / \mathrm{kg}$ de poisson. À titre de comparaison, les systèmes aquacoles conventionnels consomment de 3000 à $45000 \mathrm{~L} / \mathrm{kg}$ de poisson (Verdegem et al., 2006 ; Tal et al., 2009 ; Timmons et Ebeling, 2010) et la production d'un kilo de viande de bœuf $13500 \mathrm{~L}$. Du fait de leur faible niveau de consommation en eau, les RAS peuvent être implantés sur des sites impropres à la pratique d'activités agricoles : zones désertiques ou urbaines, permettant de se rapprocher des marchés et de réduire les frais de transport des intrants et des extrants. En maintenant en permanence le milieu d'élevage dans des conditions optimales pour les poissons d'élevage, le niveau d'efficience de l'aliment se trouve notablement amélioré et l'augmentation des densités d'élevage est rendue possible. Le recours à l'ozone et à des lampes ultraviolettes permet de neutraliser de nombreux pathogènes (bactéries, virus, champignons, protozoaires) avant que l'eau ne soit réinjectée dans le circuit ou ne soit évacuée à l'extérieur.

Les principales contraintes pour le développement à grande échelle des RAS sont le coût élevé de l'aliment, le niveau de technicité requis et la consommation d'énergie. Malgré l'utilisation d'aliments performants et à haut niveau d'efficience, les systèmes en eau recirculée peuvent voir dans certains cas s'accumuler dans les circuits certains contaminants ayant pour effet l'apparition de pathologies avec des mortalités induites. Les moyens d'améliorer ces systèmes consistent à utiliser des sources alternatives d'énergie (solaire, éolienne) et à éliminer les fines particules solides qui ont pour effet de réduire l'efficacité du processus de nitrification/ dénitrification.

\section{Situation internationale des actions menées pour affronter le changement climatique}

Dans le cadre de la mise en œuvre de son Code de conduite pour une pêche responsable (1995), la FAO (2016) a réalisé une enquête auprès de ses membres, relative aux questions de gouvernance liées aux mesures prises pour permettre à l'aquaculture de faire face aux conséquences du changement climatique (Tab. 3).

On peut constater que le zonage des sites aquacoles est globalement faible, particulièrement dans les régions où l'aquaculture est en émergence. Or, il s'agit là de la mesure la plus déterminante pour réduire la vulnérabilité de l'activité face au changement climatique. Jusqu'à présent, la répartition spatiale des infrastructures aquacoles a principalement pris en compte la disponibilité en foncier et en eau au détriment de la protection des sites. Deux mesures additionnelles, l'assistance des gouvernements en cas d'accidents climatiques et l'accès des opérateurs aquacoles aux assurances privées, sont particulièrement limitées en Asie, la région la plus vulnérable et celle qui contribue le plus largement à la production mondiale.

En matière de gestion des risques pathologiques, le score global apparaît plus élevé que pour les autres mesures prises pour faire face au changement climatique. Cependant, l'impact du changement climatique risquant d'accroître considérable- ment la fréquence et l'intensité des pathologies, une attention toute particulière devra être portée à ce secteur, notamment en Asie.

L'accès au crédit institutionnel, très peu développé, constitue une contrainte majeure pour permettre à des opérateurs aquacoles de petite et moyenne dimension de reconfigurer leurs infrastructures pour résister au changement climatique.

La prise en compte de la protection des écosystèmes enregistre un score très faible, ce qui met en évidence la sousestimation des services écosystémiques susceptibles d'assurer une protection efficace face aux impacts du changement climatique (telles que les mangroves dans le cas de l'aquaculture côtière).

Les bonnes pratiques aquacoles présentent quant à elles des bons scores et sont de bon augure pour assurer aux systèmes aquacoles un bon niveau de résilience.

\section{Conclusion : les mesures envisageables}

Face aux défis posés par le changement climatique au développement de l'aquaculture, un certain nombre de mesures, relevant de l'échelle locale, nationale et globale peut être envisagé pour réagir de façon proactive aux effets (brutaux ou à long terme) du changement climatique :

- un zonage des sites aquacoles pour minimiser les risques et une relocalisation des exploitations implantées sur des sites exposés ;

- une organisation appropriée de systèmes de veille et d'intervention et notamment dans le domaine de la pathologie des animaux aquatiques;

- l'accroissement de l'efficience de l'utilisation de l'eau par des systèmes de recyclage appropriés ;

- l'accroissement de l'efficience alimentaire pour réduire la pression sur les aliments et leurs ingrédients, notamment ceux d'origine marine ;

- la sélection et le développement d'espèces et de souches tolérantes à des facteurs du milieu susceptibles de varier avec le changement climatique, tels que la salinité, la température, l'oxygène dissous, le $\mathrm{pH}$;

- la promotion d'écloseries susceptibles d'assurer une large dissémination de ces souches et espèces ;

- la consolidation des structures d'élevage comprenant le renforcement de l'architecture des cages flottantes, l'ajustement de leur positionnement en profondeur, le rehaussement des digues et l'augmentation de la profondeur des étangs et l'adaptation des systèmes d'élevage en général pour leur permettre de faire face aux multiples agressions de l'environnement aquatique perturbé par le changement climatique.

Il convient d'insister sur l'importance du suivi local des paramètres de l'environnement, dans la mesure où l'aquaculture est extrêmement sensible aux variations, tant soudaines qu'à long terme, des conditions climatiques. Sans aller jusqu'aux systèmes automatisés de monitoring de la qualité de l'eau adoptés par de grandes fermes industrielles, des systèmes permanents de suivi simples et peu coûteux peuvent permettre d'enregistrer, d'analyser et de comprendre les causes des changements physicochimiques et biologiques du milieu 
Tableau 3. Scores moyens obtenus par divers pays en réponse à un questionnaire adressé par la FAO dans le cadre de la mise en œuvre du code pour une pêche responsable en 2015 concernant l'existence et l'application de mesures pour réduire la vulnérabilité au changement climatique (FAO, 2016).

Table 3. Average scores in the 2015 code questionnaire on aquaculture on the presence of measures for reducing vulnerability to climate change (FAO, 2016).

\begin{tabular}{|c|c|c|c|c|c|c|c|c|}
\hline Zone géographique & Afrique & Asie & Europe & $\begin{array}{l}\text { Amérique } \\
\text { latine }+ \\
\text { Caraïbe }\end{array}$ & $\begin{array}{l}\text { Proche } \\
\text { Orient }\end{array}$ & $\begin{array}{l}\text { Amérique } \\
\text { du Nord }\end{array}$ & $\begin{array}{l}\text { Pacifique } \\
\text { S-W }\end{array}$ & Global \\
\hline \multicolumn{9}{|c|}{ Mesures institutionnelles destinées à affronter le changement climatique } \\
\hline Préparation générale à gérer les risques liés au CC & 1,7 & 2,7 & 2,9 & 1,6 & 2,6 & 3,5 & 3,0 & 2,3 \\
\hline Préparation générale à faire face aux catastrophes & 2,2 & 2,9 & 3,1 & 2,2 & 2,6 & 4,0 & 3,0 & 2,6 \\
\hline $\begin{array}{l}\text { Les exploitations aquacoles bénéficient d'une assistance } \\
\text { gouvernementale en cas de catastrophe }\end{array}$ & 2,3 & 1,9 & 1,1 & 1,3 & 2,0 & 0 & 1,5 & 1,2 \\
\hline Les aquaculteurs ont accès aux assurances privées & 1,3 & 1,3 & 1,1 & 1,3 & 0,3 & 0 & 1,0 & 0,8 \\
\hline Un système de gestion des risques sanitaires est en place & 2,7 & 3,5 & 4,0 & 3,2 & 3,2 & 4,5 & 3,5 & 3,3 \\
\hline \multicolumn{9}{|c|}{ Mesures liées à la gouvernance destinées à résoudre les problèmes posés par les effets du changement climatique } \\
\hline $\begin{array}{l}\text { L'aquaculture est intégrée aux plans d'aménagement des } \\
\text { bassins versants et d'occupation des sols }\end{array}$ & 2,4 & 3,3 & 2,9 & 2,1 & 3,6 & 3,5 & 2,0 & 2,5 \\
\hline $\begin{array}{l}\text { Les fonctions écosystémiques sont prises en compte dans } \\
\text { la planification et le développement de l'aquaculture }\end{array}$ & 2,4 & 3,8 & 3,6 & 2,6 & 2,4 & 4,0 & 3,0 & 2,9 \\
\hline $\begin{array}{l}\text { Des incitations sont prévues pour la réhabilitation des } \\
\text { services écosystémiques par les aquaculteurs }\end{array}$ & 1,8 & 2,7 & 1,7 & 1,8 & 2,0 & 4,0 & 3,0 & 1,5 \\
\hline Bonnes pratiques aquacoles en place & 2,5 & 4,0 & 3,0 & 3,0 & 2,8 & 4,5 & 3,0 & 3,0 \\
\hline
\end{tabular}

$\mathrm{CC}$ : changement climatique ; le score pour chaque mesure va de 0 (mesure absente) à 5 (mesure en place, implantée et effective au niveau du terrain sur le plan national).

d'élevage et d'anticiper les événements néfastes. Cela passe par un travail de sensibilisation et de formation des aquaculteurs de la part des diverses parties prenantes, publiques et privées, de la filière aquacole.

Remerciements. L'auteur adresse ses profonds remerciements aux trois évaluateurs anonymes qui ont permis d'améliorer très significativement la qualité, sur le fond et sur la forme, de cet article.

\section{Références}

Allison EH, Perry AL, Badjeck M-C., Adger WN, Brown K, Conway $\mathrm{D}$, et al. 2009. Vulnerability of national economies to the impacts of climate change on fisheries. Fish and Fisheries 10: 173-196.

Alric B. 2006. L'influence des perturbations locales sur la vulnérabilité et la réponse des réseaux trophiques pélagiques lacustres face au changement climatique. Thèse de doctorat, université de Grenoble (France).

Anh NL. 2014. Climate proofing aquaculture: a case study on pangasius farming in the Mekong Delta, Vietnam. $\mathrm{PhD}$ thesis, Wageningen University (Wageningen [NL]).
Arias-Schreiber M, Niquen M, Bouchon M. 2011. Coping strategies to deal with environmental variability and extreme climatic events in the Peruvian Anchovy Fishery. Sustainability 3: 823-846.

Asmah R, Karikari A, Al-Shihi J, Handisyde N, Xia F, Telfer T, et al. 2015. Spatial models for optimization of zones for improved and sustainable cage aquaculture in Volta Lake, Ghana. In: Aquaculture Europe 2015, Rotterdam, Netherlands. Available from: https:// www.was.org/easOnline/AbstractDetail.aspx? $\mathrm{i}=4115$.

Blanchard JL, Jennings S, Holmes R, Harle J, Merino G, Allen JI, et al. 2012. Potential consequences of climate change for primary production and fish production in large marine ecosystems. Philosophical Transactions of the Royal Society B 367(1605): 2979-2989. doi:10.1098/rstb.2012.0231.

Cacot P, Lazard J. 2009. La domestication des poissons du Mékong : les enjeux et le potentiel aquacole. Cah Agric 18 (2-3): 125-135.

Castaneda R, McGee M, Velasco M. 2010. Pangasius juveniles tolerate moderate salinity in test. Global Aquaculture Advocate: 27-28.

Clota F. 2006. Rapport de mission au Laos. Available from http://umrintrepid.cirad.fr/content/download/4361/32542/version/1/file/ F.+CLOTA, +CIRAD, rapport+de+mission+Laos+2006.pdf, last consult: 10/2016.

De Silva SS, Soto D. 2009. Climate change and aquaculture: potential impacts, adaptation and mitigation. In: Cochrane K, De Young C, 
Soto D, Bahri T, eds. Climate change implications for fisheries and aquaculture: overview of current scientific knowledge. FAO Fisheries and Aquaculture Technical Paper No. 530. Rome: FAO, pp. 156-212.

De Silva SS, Phuong TN. 2011. Striped catfish farming in the Mekong Delta: a tumultuous path to a global success. Reviews in Aquaculture 3: 45-73

De Silva SS. 2012. Climate change impacts: challenges for aquaculture. In: Subasinghe RP, Arthur JR, Bartley DM, De Silva SS, Halwart M, Hishamunda N, et al., eds. In: Farming the Waters for People and Food. Proceedings of the Global Conference on Aquaculture 2010, Phuket, Thailand. Rome: FAO and Bangkok: NACA, pp. 75-110.

Do TTH, Tran NTQ. 2012. The effect of salinity on the embryonic development and osmoregulatory of the striped catfish (Pangasianodon hypophthalmus) larvae and fingerling stages. Journal of Science 21b: 29-37, Can Tho University, Vietnam, in Vietnamese with English summary.

Falkernmark M, Rockstöm J, Karlberg L. 2009. Present and future water requirements for feeding humanity. Food Security 1: 59-69.

FAO. 2014. The state of world fisheries and aquaculture 2014. Contributing to food security and nutrition for all. Rome: FAO.

FAO. 2016. The state of world fisheries and aquaculture 2016. Contributing to food security and nutrition for all. Rome: FAO.

Ficke AD, Myrick CA, Hansen LJ. 2007. Potential impacts of global climate change on freshwater fisheries. Reviews in Fish Biology and Fisheries 17: 581-613.

Fish Farmer. 2008. Norwegian study on impact of climate change on farmed salmon. p. 1. Available from www.fishfarmer-magazine. com/news/fullstory.php/aid/1490/.

Grinsted A, Moore JC, Jevrejeva S. 2009. Reconstructing sea level from paleo and projected temperatures 200 to 2100 AD. Climate Dynamics 34: 461. doi: 10.1007/s00382-008-0507-2.

Gros P. 2014. La pêche, une activité ancestrale en mutation. Cah Agric 23: 4-17.

Gruber N, Hauri C, Lachkar Z, Loher D, Frölicher TL, Plattner GK. 2012. Rapid progression of ocean acidification in the California current system. Science 337: 220-223. doi: 10.1126/ science. 1216773 .

Halls AS, Johns M. 2013. Assessment of the vulnerability of the Mekong Delta Pangasius catfish industry to development and climate change in the Lower Mekong Basin. Report prepared for the Sustainable Fisheries Partnership. Bath, UK: Johns Associates Limited.

IPCC. 2014. Climate change 2014: synthesis report. Available from https://www.ipcc.ch/report/ar5/syr/.

Kayansamruaj P, Pirarat N, Hirono I, Rodkhum C. 2014. Increasing of temperature induces pathogenicity of Streptococcus agalactiae and the up-regulation of inflammatory related genes in infected Nile tilapia (Oreochromis niloticus). Veterinary microbiology 172 (1): 265-271.

Keeling RF, Körtzinger A, Gruber N. 2010. Ocean deoxygenation in a warming world. Annual Review of Marine Science 2: 199-229. doi: 10.1146/annurev.marine.010908.163855.

Klinger D, Naylor R. 2012. Searching for solutions in aquaculture: charting a sustainable course. Annu Rev Environ Resour 37:247276. doi: 10.1146/annurev-environ-021111-161531.

Lazard J, Cacot P, Slembrouck J, Legendre M. 2009. La pisciculture des Pangasiidae. Cah Agric 18 (2-3): 164-173.

Lazard J, Rey-Valette H, Aubin J, Mathé S, Chia E, Caruso D, et al. 2014. Assessing aquaculture sustainability: a comparative methodology. International Journal of Sustainable Development \& World Ecology 21(6): 503-511. doi: 10.1080/13504509.2014.964350.
Lazzaro X, Starling F. 2005 Using biomanipulation to control eutrophication in a shallow tropical urban reservoir (Lago Paranoa, Brazil). Restoration and Management of Tropical Eutrophic Lakes: 361-387.

Levitus S, Antonov JI, Boyer TP, Baranova OK, Garcia HE, Locarnini RA, et al. 2012. World ocean heat content and thermosteric sea level change (0-2000 m), 1955-2010. Geophysical Research Letters 39: L10603. doi:10.1029/2012GL051106.

Marcusso PF, Aguinaga JY, Claudiano GdS, Eto SF, Fernandes DC, Mello H, et al. 2015. Influence of temperature on Streptococcus agalactiae infection in Nile tilapia. Braz J Vet Res Anim Sci 52(1): $57-62$.

Médale F, Le Boucher R, Dupont-Nivet M, Quillet E, Aubin J, Panserat $\mathrm{S}$. 2013. Des aliments à base de végétaux pour les poissons d'élevage. Inra Prod Anim 26(4): 303-316.

Merino M, Barange M, Blanchard JL, Harle J, Holmes R, Allen I, et al. 2012. Can marine fisheries and aquaculture meet fish demand from a growing human population in a changing climate? Global Environmental Change 22(4): 795-806. doi: 10.1016/j.gloenv cha.2012.03.003.

Nguyen TTT, De Silva SS. 2006. Fresh water finfish biodiversity and conservation: an Asian perspective. Biodiversity and Conservation 15: 3543-3568.

Nguyen CL, Do TTH, Huong VNS, Nguyen TP. 2011. Physiological changes and growth of the striped catfish (Pangasianodon hypophthalmus) exposed to different salinities. Journal of Science 17a: 60-69, Can Tho University, Vietnam, in Vietnamese, English summary.

Sabine CL, Tanhua T. 2010. Estimation of anthropogenic $\mathrm{CO}_{2}$ inventories in the ocean. Annual Review of Marine Science 2: 175-198. doi: 10.1146/annurev-marine-120308-080947.

Seibel BA. 2011. Critical oxygen levels and metabolic suppression in oceanic oxygen minimum zones. Journal of Experimental Biology 214(2): 326-336. doi: 10.1242/jeb.049171.

Smith ADM, Brown CJ, Bulman CM, Fulton EA, Johnson P, Kaplan IC, et al. 2011. Impacts of fishing low-trophic level species on marine ecosystems. Science 333: 1147-1150.

Spataru P, Gophen M. 1985. Feeding behaviour of silver carp Hypophthalmichthys molitrix Val. and its impact on the food web in Lake Kinneret, Israel. Hydrobiologia 120: 53. doi:10.1007/ BF00034590.

Tacon AGJ, Metian M. 2015. Feed matters: satisfying the feed demand of aquaculture. Reviews in Fisheries Science \& Aquaculture 23: $1-10$.

Tacon AGJ, Metian M, Turchini GM, De Silva SS. 2010. Responsible aquaculture and trophic level implications to global fish supply. Reviews in Fisheries Science 18 (1): 94-105.

Tacon AGJ, Hasan MR, Metian M. 2011. Demand and supply of feed ingredients for farmed fish and crustaceans: trends and prospects. FAO Fisheries and Aquaculture Technical Paper No. 564. Rome: FAO.

Tal Y, Schreier HJ, Sowers KR, Stubblefield JD, Place AR. 2009. Environmentally sustainable land based marine aquaculture. Aquaculture 286: 28-35.

Tan XH. 2008. Study on climate change scenarios assessment for Ca Mau Province. Technical report. Ho Chi Minh City (Vietnam): Southern Institute for Water Resources Planning.

Thackery SJ, Sparks TH, Frederiksen M, Burthe S, Bacon PJ, Bell JR, et al. 2010. Trophic level asynchrony in rates of phenological change of marine, freshwater and terrestrial environments. Global Change Biology 16: 3304-3313.

Timmons MB, Ebeling JM. 2010. Recirculating aquaculture. Ithaca, NY: Cayuga Aqua Ventures. 
Travers M-A., Basuyaux O, Le Goic N, Huchette S, Nicolas J-L., Koken $\mathrm{M}$, et al. 2009. Influence of temperature and spawning effort on Haliotis tuberculata mortalities caused by Vibrio harveyi: an example of emerging vibriosis linked to global warming. Global Change Biology 15: 1365-1376.

Tyedmers P, Pelletier N. 2007. Biophysical accounting in aquaculture: insights from current practice and the need for methodological development. In: Bartley DM, Brugre C, Soto D, Gerber P, Harvey B, eds. Comparative assessment of the environment costs of aquaculture and other food production sectors: methods of meaningful comparisons. FAO Fisheries Proceedings No. 10. Rome: FAO, pp. 229-241.

Vass KK, Das MK, Srivastava PK, Dey S. 2009. Assessing the impact of climate change on inland fisheries in River Ganga and the plains in India. Aquatic Ecosystem Health \& Management 2: 138-151.

Verdegem MCJ, Bosma RH, Verreth JAJ. 2006. Reducing water use for animal production through aquaculture. Int J Water Resour Dev 22: $101-113$.

Citation de l'article : Lazard J. 2017. Les systèmes aquacoles face au changement climatique. Cah. Agric. $26: 34001$. 